\title{
Erratum: A genome-wide association study of cleft lip with and without cleft palate identifies risk variants near MAFB and $A B C A 4$
}

Terri H Beaty, Jeffrey C Murray, Mary L Marazita, Ronald G Munger, Ingo Ruczinski Jacqueline B Hetmanski, Kung Yee Liang, Tao Wu, Tanda Murray, M Daniele Fallin, Richard A Redett, Gerald Raymond, Holger Schwender, Shin C Jin, Margaret E Cooper, Martine Dunnwald, Maria A Mansilla, Elizabeth Leslie, Stephen Bullard, Andrew C Lidral, Lina M Moreno, Renato Menezes, Alexandre R Vieira, Aline Petrin, Allen J Wilcox, Rolv T Lie, Ethylin W Jabs, Yah Huei Wu-Chou, Philip K Chen, Hong Wang, Xiaoqian Ye, Shangzhi Huang, Vincent Yeow, Samuel S Chong, Sun Ha Jee, Bing Shi, Kaare Christensen, Mads Melbye, Kimberly F Doheny, Elizabeth W Pugh, Hua Ling, Eduardo E Castilla, Andrew E Czeizel, Lian Ma, L Leigh Field, Lawrence Brody, Faith Pangilinan, James L Mills, Anne M Molloy, Peadar N Kirke, James M Scott, Mauricio Arcos-Burgos \& Alan F Scott Nat. Genet. 42, 525-529 (2010); published online 2 May 2010; corrected after print 12 July 2010.

In the version of this article initially published, the author John M Scott (Trinity College, Dublin) was incorrectly written as James M Scott. The error has been corrected in the HTML and PDF versions of the article. 